\title{
Aneurisma da Veia Jugular Externa Trombosado: Causa Rara de Tumefação Cervical
}
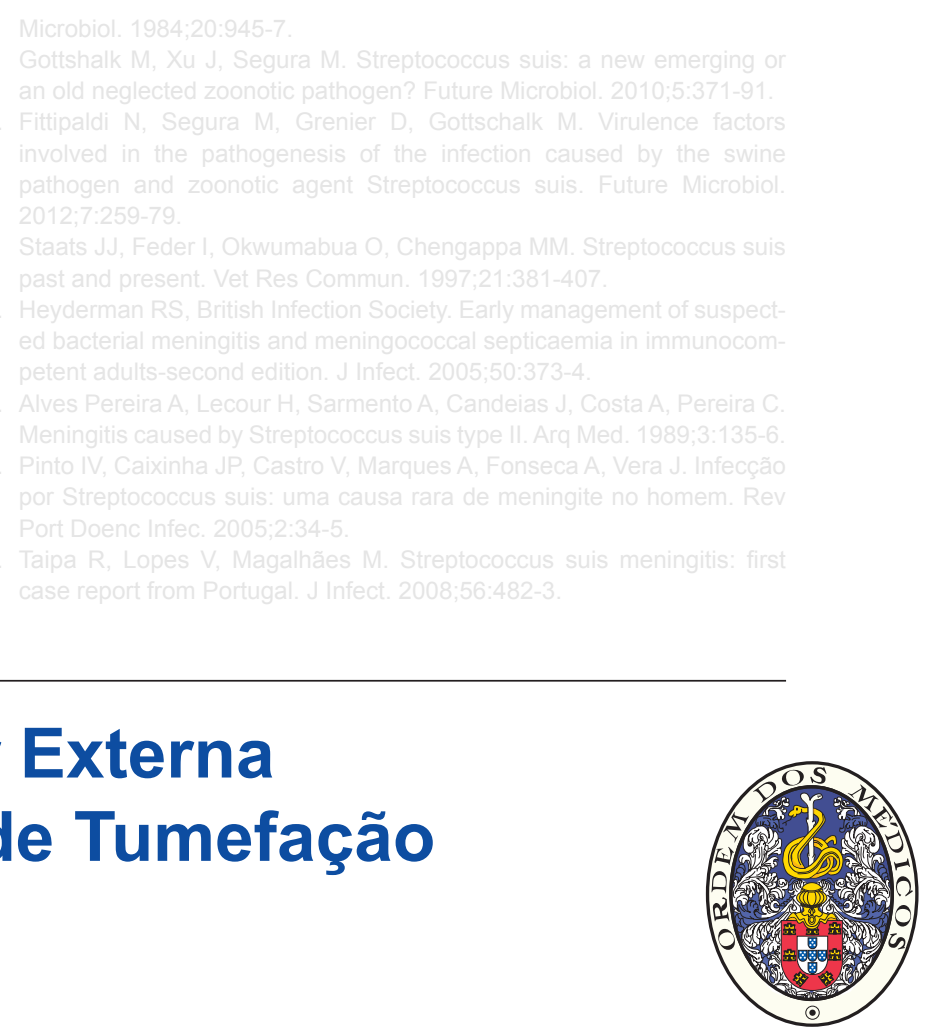

\section{Thrombosed External Jugular Vein Aneurysm: a Rare Cause of Neck Mass}

Inês Santiago MARTINS ${ }^{1}$, Hugo Pisco PACHECO ${ }^{1}$, Isabel SAPEIRA ${ }^{1}$ Acta Med Port 2014 Jul-Aug;27(4):521-524

\section{RESUMO}

Os aneurismas venosos raramente são responsáveis por tumefações cervicais, e em relação às veias do pescoço, os aneurismas da veia jugular externa são extremamente raros, sendo poucos os casos descritos na literatura. Embora na maioria assintomáticos, podem causar dor, complicar com trombose ou mesmo rotura. Os exames de imagem têm papel no diagnóstico e no planeamento cirúrgico desta patologia. Enfatizando-se o contributo dos meios de imagem, os autores apresentam o caso de um aneurisma da veia jugular externa, inicialmente assintomático mas que posteriormente se complicou por trombose.

Palavras-chave: Veias Jugulares; Aneurisma; Trombose; Tomografia Computorizada; Ecografia.

\section{ABSTRACT}

Venous aneurysms are rarely responsible for neck masses, and regarding the neck veins, external jugular vein aneurysms are extremely uncommon, with a few cases reported in the literature. Although most cases are asymptomatic, they may cause pain, be complicated by thrombosis or even rupture. Imaging studies have a role in the diagnosis and surgical planning of this pathology. With emphasis on the

1. Serviço de Imagiologia. Hospital Distrital de Santarém. Santarém. Portugal.

Recebido: 29 de Novembro de 2013 - Aceite: 01 de Março de 2014 | Copyright @ Ordem dos Médicos 2014 
imaging techniques, the authors present a case of an external jugular vein aneurysm, initially asymptomatic but thereafter complicated with thrombosis.

Keywords: Jugular Veins; Aneurysm; Thrombosis; Tomography, X-Ray Computed; Ultrasonography.

\section{INTRODUÇÂO}

Os aneurismas venosos são dilatações fusiformes ou saculares quase sempre solitárias, que comunicam com a veia originária por um único canal, denominado colo. As dilatações aneurismáticas nas veias do pescoço são raras, devido à baixa pressão do sistema da veia cava. Relativamente às veias do pescoço, os aneurismas da veia jugular externa são bem mais raros que os da veia jugular interna, segundo os trabalhos até à data publicados, a maioria casos clínicos isolados. ${ }^{1-11}$

\section{CASO CLÍNICO}

Doente do sexo feminino, 68 anos, com hipertensão arterial controlada, sem outros antecedentes clínicos relevantes nomeadamente fatores de risco cardiovasculares como diabetes, dislipidemia ou tabagismo. Clinicamente, observava-se uma tumefação cervical direita com anos de evolução, previamente caracterizada como ectasia da veia jugular externa. Recentemente, a doente referia odinofagia, sendo referenciada ao nosso serviço para realização de Angio-Tomografia Computorizada (Angio-TC) dos vasos supra-aórticos para melhor caracterização da ectasia da veia jugular externa direita e a relação com as estrutu- ras adjacentes. No referido exame, observámos dilatação aneurismática sacular da veia jugular externa direita, inferiormente à parótida, com 7,2 x 4,3 x 2,9 cm, sem outras alterações imagiológicas assinaláveis, nomeadamente sem compressão de estruturas adjacentes como o esófago ou traqueia (Fig. 1).

Seis meses depois, a doente recorreu ao Serviço de Urgência do nosso hospital referindo aumento dimensional da referida lesão cervical, associada a dor e hipertermia local. Realizou-se Eco-Doppler dos vasos do pescoço que evidenciou no interior da dilatação aneurismática da veia jugular externa direita imagem ecogénica mista com $4,3 \mathrm{~cm}$ de maior eixo, compatível com trombo, parcialmente oclusivo (Fig. 2).

A doente foi submetida a cirurgia de excisão total do aneurisma da veia jugular externa direita, mediante laqueação distal e proximal do vaso. O procedimento decorreu sem intercorrências, com evolução favorável um ano após a cirurgia. $O$ estudo anátomo-patológico da peça operatória confirmou aneurisma venoso com $4,5 \mathrm{~cm}$ de comprimento e com um diâmetro máximo de $4 \mathrm{~cm}$, preenchido parcialmente por coágulo.

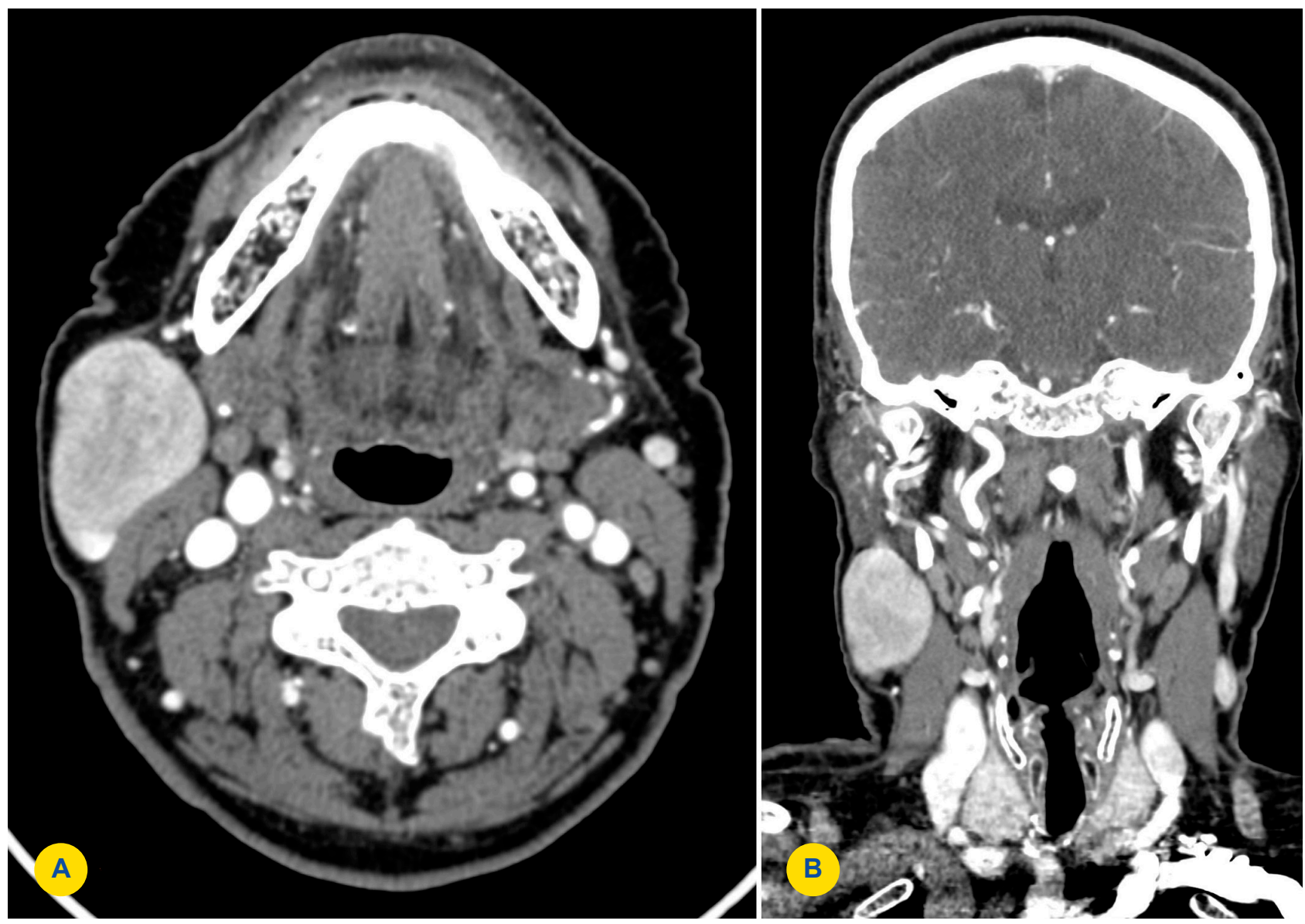

Figura 1 - Aneurisma sacular da veia jugular externa direita. Angio-Tomografia Computorizada dos vasos do pescoço nos planos axil (A), coronal (B) 

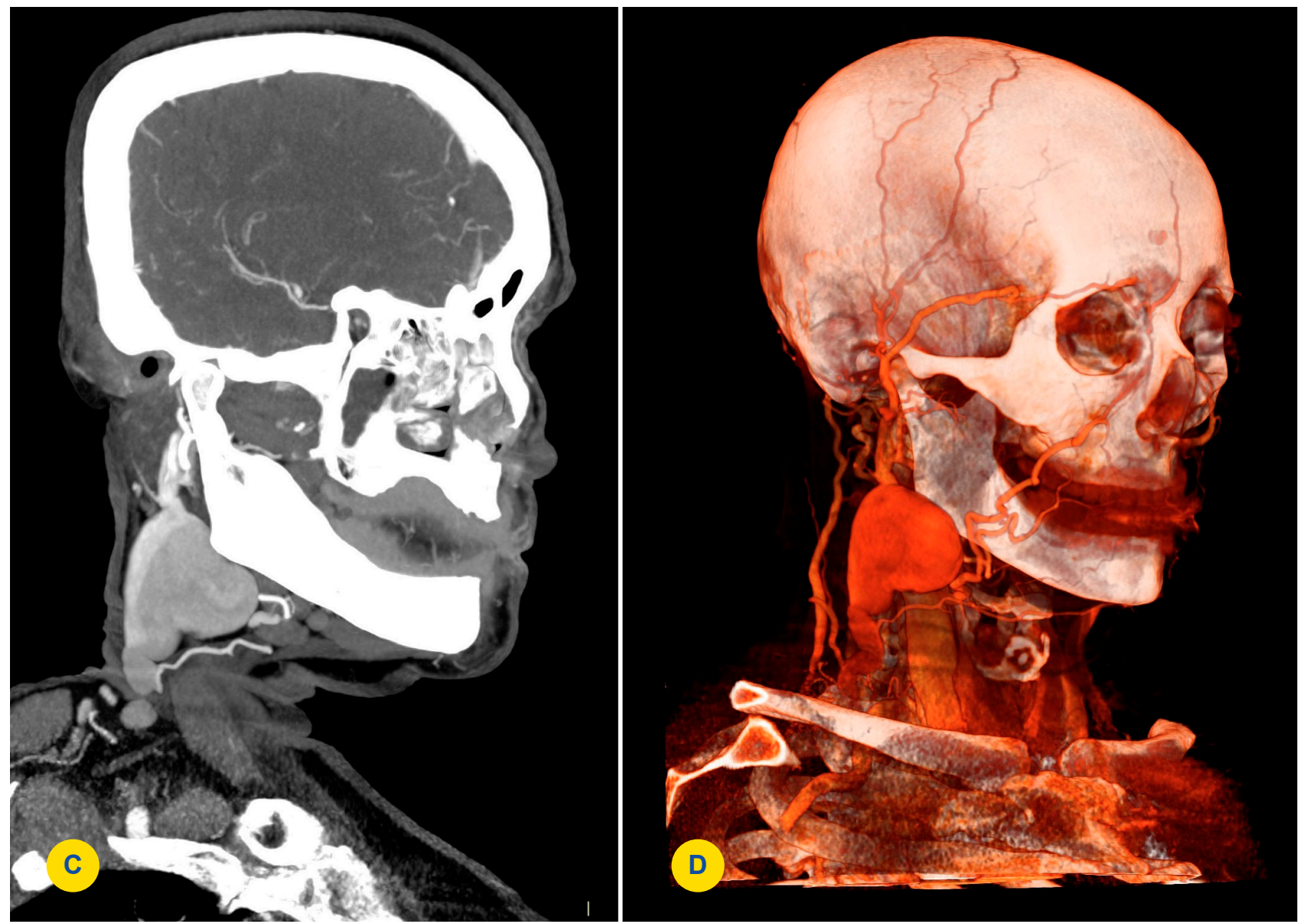

Figura 1 - Aneurisma sacular da veia jugular externa direita. Angio-Tomografia Computorizada dos vasos do pescoço nos planos sagital oblíquo (C) e imagem de reconstrução pós-processada em volume rendering (D).

\section{DISCUSSÃo}

Os aneurismas venosos são dilatações fusiformes ou saculares geralmente solitárias, que comunicam com a veia originária por um colo, sendo entidades distintas das veias varicosas (fleboectasias), pseudoaneurismas ou fístulas arteriovenosas, e não associadas a alterações hemodinâmicas. ${ }^{1,2,12} \mathrm{~A}$ sua fisiopatologia ainda é desconhecida. Os aneurismas venosos podem ser classificados em: primários/congénitos ou secundários a alterações traumáticas, inflamatórias ou tumores, podendo ainda surgir espontaneamente. ${ }^{3-5}$ Foi recentemente descrito um caso relacionado com a presença de uma anomalia valvular venosa. ${ }^{8}$ Não está documentada associação com idade ou sexo. ${ }^{1,2,12}$ Qualquer veia pode ser afectada, incluindo as veias do pescoço (do sistema venoso superficial e profundo), veias torácicas, viscerais e dos membros inferiores. ${ }^{3}$

As dilatações aneurismáticas das veias do pescoço são raras, pela baixa pressão no sistema da veia cava superior, ${ }^{3}$ sendo, entre estas veias, observadas sobretudo na veia jugular interna. ${ }^{4,6}$ Os aneurismas da veia jugular externa são ainda mais infrequentes, em especial os de morfologia sacular, como no presente caso. ${ }^{2,4,5,12}$

A apresentação clínica consiste habitualmente em tumefação cervical assintomática, podendo gradualmente aumentar de dimensões. Por vezes, estes aneurismas podem causar dor e desconforto, mas geralmente têm uma

\section{evolução benigna. $2,4,5,12$}

Embora na maioria dos casos os aneurismas venosos cervicais superficiais do pescoço sejam assintomáticos, podem ocorrer complicações, como trombose, tromboflebite e rotura, ${ }^{2,3,5}$ tendo sido descrito um caso de trombose recorrente. ${ }^{8} \mathrm{~A}$ trombose venosa superficial raramente cursa com tromboembolismo pulmonar devido à natureza aderente deste tipo de trombos. Ainda assim, foi recentemente descrito um caso de um aneurisma trombosado da veia jugular externa como causa de tromboembolismo pulmonar. ${ }^{1}$ Os aneurismas podem também ser causa de compressão de estruturas nervosas adjacentes e cursar com parestesias. ${ }^{8}$

Os diagnósticos clínicos diferenciais mais comuns incluem: adenopatias, patologia tiroideia, quistos cervicais congénitos (do aparelho branqueal e outros), linfocelo, laringocelo, lipoma, malformações arteriovenosas e aneurismas arteriais, sendo de considerar nas crianças a possibilidade de higroma quístico e hemangioma cavernoso. .-6,12,13 $^{-1}$ O diagnóstico pode ser sugerido clinicamente, devendo ser confirmado por métodos de imagem. ${ }^{4} \mathrm{O}$ aumento da lesão com a tosse, esforço ou manobra de Valsalva sugere o diagnóstico de ectasia ou aneurisma da veia jugular. ${ }^{12,13}$

$\mathrm{Na}$ fase de avaliação seguinte, a ecografia com estudo Doppler é a técnica de eleição devido ao baixo custo e boa acuidade diagnóstica. A ecografia permite primeiramente o diagnóstico diferencial entre lesões quísticas e sólidas, 


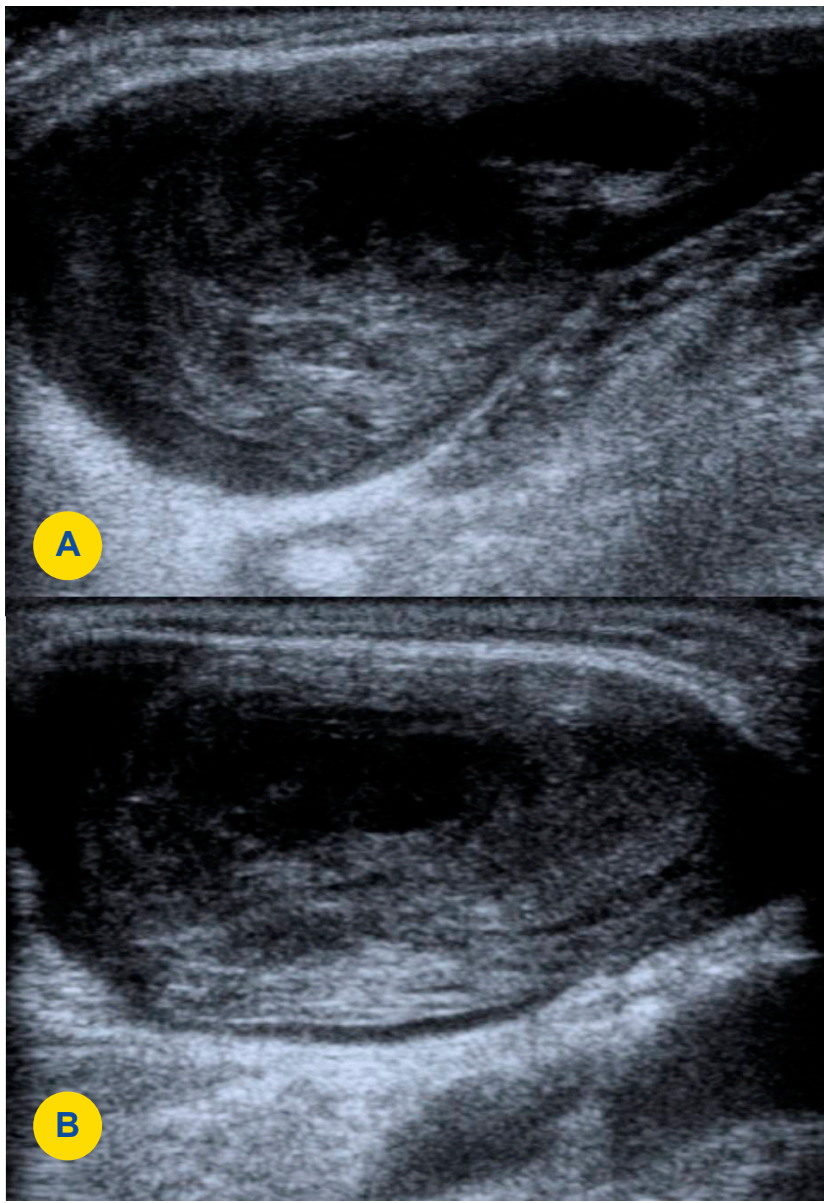

Figura 2 - Ecografia convencional, evidenciando trombo no interior da dilatação aneurismática da veia jugular externa direita, em plano longitudinal $(A)$ e transversal $(B)$

estabelecer a origem da lesão em relação a estruturas adjacentes, e distinguir lesões vasculares de não vasculares. Quistos e laringocelos distinguem-se de lesões venosas pela ausência de fluxo no estudo Doppler ou de comunicação com o sistema vascular. Entre as lesões vasculares, os aneurismas arteriais e fístulas arteriovenosas distinguem-se de aneurismas venosos pela demonstração da comunicação com uma artéria ou entre segmentos arteriais e venosos, e utilizando o estudo Doppler, que evidencia um traçado arterial ou turbulento, respetivamente. A ecografia permite identificar o vaso de origem do aneurisma, assim como a avaliação de complicações associadas, como a trombose, sendo útil no planeamento cirúrgico. ${ }^{4,5}$

A ecografia com color-Doppler é suficiente para o diagnóstico, mas a TC e a Ressonância Magnética (RM) permitem determinar com mais precisão o tamanho e a extensão da lesão, confirmar a origem vascular e avaliar a relação e o efeito nas estruturas adjacentes. Após a administração de contraste na TC, um ligeiro aumento de densidade na fase arterial e um aumento homogéneo numa fase mais tardia. ${ }^{5,12,13}$ A RM pode também ser útil na avaliação dos aneurismas venosos, não havendo tanto quanto se sabe, descrição dos aspetos em RM deste tipo específico de aneurismas.

Na maioria dos casos, os aneurismas jugulares externos têm uma evolução benigna, optando-se por realizar seguimento de forma conservadora. ${ }^{7,13} \mathrm{~A}$ cirurgia realiza-se sobretudo por razões estéticas e na presença de sintomas. Embora não existam normas estabelecidas, alguns autores advogam o tratamento cirúrgico por excisão devido ao risco de trombose, com complicações tromboembólicas e rotura. ${ }^{2,3,5,7}$

\section{CONCLUSÃO}

Os aneurismas da veia jugular externa são raros e apesar de em geral assintomáticos, podem causar dor, ou complicar com trombose ou rotura. É importante o diagnóstico diferencial desta entidade entre outras lesões cervicais, e neste campo os exames de imagem têm um papel importante, quer na fase do diagnóstico, quer no planeamento cirúrgico.

\section{CONFLITOS DE INTERESSE}

Os autores declaram não terem quaisquer conflitos de interesse.

\section{FONTE DE FINANCIAMENTO}

Os autores declaram que para a realização do presente trabalho não existiu qualquer financiamento.

\section{REFERÊNCIAS}

1. Ioannou CV, Kostas T, Tsetis D, Georgakarakos E, Gionis M, Katsamouris AN. External jugular vein aneurysm: a source of thrombotic complications. Int Angiol. 2010;29:284-5.

2. Karapolat $S$, Erkut $B$, Ünlü Y. Multiple aneurysms of the left external jugular vein. Turk J Med Sci. 2005;35:43-5.

3. Cãdariu FI, Avram J, Enache Al, Pasztori M, Avram I, Stãnciulescu C, et al. External jugular vein aneurysm - case report. J Exp Med Surg Res. 2010;4:271-4.

4. Drakonaki EE, Symvoulakis EK, Fachouridi A, Kounalakis D, Tsafantakis E. External jugular vein aneurysm presenting as a cervical mass. Int J Otolaryngol. 2011;2011:485293.

5. Lee HY, Lee W, Cho YK, Chung JW, Park JH. Superficial venous aneurysm: reports of 3 cases and literature review. J Ultrasound Med. 2006;25:771-6.

6. Verma RK, Kaushal D, Panda NK. External jugular vein aneurysm with thrombus presenting as painful neck mass: a case report. Oman Med J. 2013;28:278-80.

7. Mohanty D, Jain BK, Garg PK, Tandon A. External jugular venous aneu- rysm: A clinical curiosity. J Nat Sci Biol Med. 2013;4:223-5

8. Gianesini S, Menegatti E, Zuolo M, Occhionorelli S, Ascanelli S, Zamboni P. Spontaneous thrombosis of primary external jugular veins aneurysms. Veins Lymphatics. 2013;1:e17:60-2.

9. Rawat NS, Gupta A, Khurana P, Jain S, Trehan N. MSCT angiography diagnosis of thrombosis in external jugular venous aneurysm: case report and review of literature. Ind Heart J. 2008;60:52-4.

10. Verbeeck N, Hammer F, Goffette P, Mathurin P. Saccular aneurysm of the external jugular vein, an unusual cause of neck swelling. J Belge Radiol. 1997;80:63-4.

11. Kirmani S, Rashid M, Ali I, Badar F. External jugular vein aneurysm: a rare cause of neck swelling. J Ultrasound Med. 2011;30:1157-8.

12. Ilijevski NS, Radak S, Vucurević G, Sagić D, Otasević P, Tasić N, et al. Jugular vein aneurysm. Vascular. 2008;16:291-4.

13. Lee HY, Yoo SM, Song IS, Yu H, Lee JB. Sonographic diagnosis of a saccular aneurysm of the internal jugular vein. J Clin Ultrasound. 2007;35:94-6 


\section{Aneurisma da Veia Jugular Externa Trombosado: Causa Rara de Tumefação Cervical

\author{
Acta Med Port 2014:27:521-524
}

Publicado pela Acta Médica Portuguesa, a Revista Científica da Ordem dos Médicos

Av. Almirante Gago Coutinho, 151

1749-084 Lisboa, Portugal.

Tel: +351218428 215

E-mail: submissao@actamedicaportuguesa.com

www.actamedicaportuguesa.com

ISSN:0870-399X | e-ISSN: 1646-0758

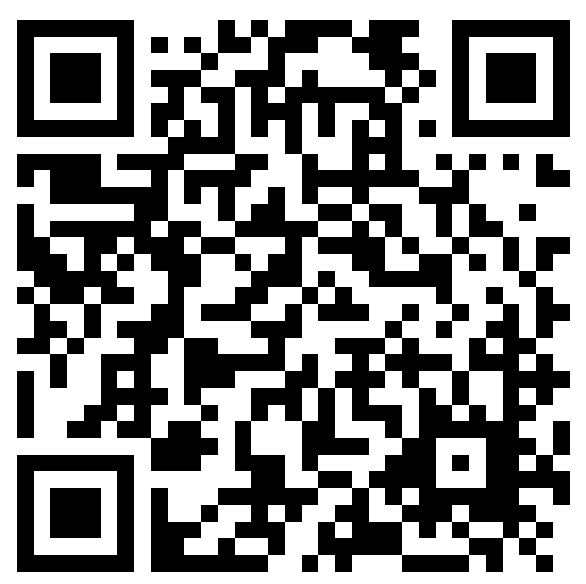

